\title{
The role of the proteasome in the generation of MHC class I ligands and immune responses
}

\author{
E. J. A. M. Sijts · P.-M. Kloetzel
}

Received: 17 February 2011/Revised: 17 February 2011/Accepted: 18 February 2011/Published online: 9 March 2011

(C) The Author(s) 2011. This article is published with open access at Springerlink.com

\begin{abstract}
The ubiquitin-proteasome system (UPS) degrades intracellular proteins into peptide fragments that can be presented by major histocompatibility complex (MHC) class I molecules. While the UPS is functional in all mammalian cells, its subunit composition differs depending on cell type and stimuli received. Thus, cells of the hematopoietic lineage and cells exposed to (pro)inflammatory cytokines express three proteasome immunosubunits, which form the catalytic centers of immunoproteasomes, and the proteasome activator PA28. Cortical thymic epithelial cells express a thymus-specific proteasome subunit that induces the assembly of thymoproteasomes. We here review new developments regarding the role of these different proteasome components in MHC class I antigen processing, T cell repertoire selection and CD8 $\mathrm{T}$ cell responses. We further discuss recently discovered functions of proteasomes in peptide splicing, lymphocyte survival and the regulation of cytokine production and inflammatory responses.
\end{abstract}

Keywords Proteasome - Immunosubunits - PA28 · MHC class I - Antigen processing · Immune responses · Peptide splicing $\cdot$ TCR repertoire

\footnotetext{
E. J. A. M. Sijts

Department of Infectious Diseases and Immunology,

Faculty of Veterinary Medicine, Utrecht University,

Yalelaan 1, 3584CL Utrecht, The Netherlands

e-mail: e.j.a.m.sijts@uu.nl

P.-M. Kloetzel ( $\square)$

Institut für Biochemie, Charité-Universitätsmedizin Berlin,

Oudenarderstr. 16, 13347 Berlin, Germany

e-mail: p-m.kloetzel@charite.de
}

\section{The ubiquitin-proteasome system}

Cell-surface-expressed major histocompatibility complex (MHC) class I molecules present antigenic peptides to the outside world so that they can be specifically recognized by cytotoxic T lymphocytes (CTLs) [1]. These specialized T cells can detect other cells that endogenously express foreign or aberrant (i.e. mutated) protein molecules and subsequently remove these unwanted cells from the body. Recognition fully depends on the binding of antigenic peptides into the groove of MHC class I molecules [2, 3]. The generation of these peptides requires the degradation of proteins by the $26 \mathrm{~S}$ proteasome into peptide fragments of a size appropriate for transport by the transporter associated with antigen presentations (TAPs) into the endoplasmic reticulum (ER) or for docking to the peptide binding groove of the MHC class I molecule [4-7].

The $26 \mathrm{~S}$ proteasome is the central proteolytic enzyme complex of the ubiquitin-proteasome system (UPS) and responsible for the degradation of poly-ubiquitylated proteins formed by a cascade of E1, E2 and E3 enzymes, which activate, conjugate and transfer multiple ubiquitin (ub) moieties to protein substrates to be degraded [8]. The multiple subunit $20 \mathrm{~S}$ proteasome is the catalytic core of the $26 \mathrm{~S}$ proteasome, which is built by the association of the $20 \mathrm{~S}$ core complex with two 19S regulator complexes that are responsible for the activation of the $20 \mathrm{~S}$ core as well as for the binding and unfolding of ubiquitylated substrates [19S-20S-19S] [9, 10].

The 20S proteasome is composed of 14 non-identical subunits building four stacked rings of seven subunits each. Seven different but related $\alpha$-subunits $(\alpha 1-\alpha 7)$ form the two outer $\alpha$-rings, whereas the two inner rings contain seven different $\beta$-subunits $(\beta 1-\beta 7)[11,12]$. With its $\mathrm{N}$-terminal threonine residues as the single active site of the $\beta$-subunits 
$\beta 1, \beta 2$ and $\beta 5$, the $20 \mathrm{~S}$ proteasome is a N-terminal nucleophilic hydrolase responsible for the generation of the vast majority of virus or tumor-derived peptides presented by MHC class I molecules at the cell surface.

The three active site subunits differ in their ability to hydrolyze short fluorogenic peptide substrates. Accordingly, the activities are referred to as chymotrypsin-like $(\beta 5)$, trypsin-like $(\beta 2)$, or caspase-like $(\beta 1)$ [11]. Not neglecting certain preferences, cleavage site usage by the proteasome is promiscuous in that almost every amino acid residue can serve as a cleavage site, giving the enzyme complex a high degree of flexibility with regard to quality of the products that are generated $[7,13,14]$. The reasons why proteasomes emerged as the main protease thought to be involved in the generation of MHC class I epitope are several-fold. First of all, proteasomes preferentially cleave at the carboxy-terminus after hydrophobic or basic residues thereby preferentially generating the C-terminal anchor residue of MHC class I epitopes. Secondly, proteasomes generate peptides of 8-10 amino acids in length and thus of a size suited for binding to MHC class I molecules [15]. Furthermore, inhibition of proteasome activity using specific inhibitors abolished MHC class I antigen presentation almost completely $[4,16]$. The most profound influence on the notion that proteasomes are involved in antigen processing resulted, however, from the finding that, upon IFN- $\gamma$ induction, specific catalytically active $\beta$-subunits, i.e. $\beta 1 \mathrm{i} / \mathrm{LMP} 2, \beta 2 \mathrm{i} / \mathrm{MECL} 1$ and $\beta 5 \mathrm{i} / \mathrm{LMP} 7$, are incorporated into nascent proteasomes to form $20 \mathrm{~S}$ proteasome complexes with an alternative subunit composition [17]. Two of these subunits ( $\beta 1 \mathrm{i} / \mathrm{LMP} 2, \beta 5 \mathrm{i} / \mathrm{LMP} 7)$ are encoded within the MHC class II region [18-21], which led to the terms immunosubunits (i-subunits) and immunoproteasome (i-proteasome) [22] implying that these enzyme complexes are responsible for antigen presentation. Because of this change in active site subunits, immunoproteasomes have an altered cleavage site preference as well as a different cleavage rate [23-26]. The previously identified proteasome maturation protein is also up-regulated by IFN- and accelerates immunoproteasome formation through functional interaction with the $\beta 5$ i subunit [27].

Thus, two types of proteasomes exist, i.e. standard proteasomes that are constitutively expressed in all cells of an organism, and immunoproteasomes that are constitutively expressed in immune relevant cells and are formed upon exposure of cells to IFN- $\gamma$ or particular proinflammatory cytokines, as discussed below.

The emerging picture appeared to be complete when the proteasome activator subunits PA2 $8 \alpha$ and PA28 $\beta[28,29]$, which bind to the outer $\alpha$-rings of the 20S proteasome thereby forming so-called hybrid-proteasomes [19S-20SPA28] [30, 31], were also found to be induced by IFN- $\gamma$ [32]. Expression of PA28, however, is not restricted to
IFN- $\gamma$ induction, but is found in almost all tissues analyzed. Biochemical studies revealed that PA28 does not activate the proteasome by directly affecting the hydrolyzing activities of the different active sites buried in the catalytic cavity [33, 34]. In contrast, structural and biochemical evidence suggests that, by binding to the outer $\alpha$-rings of the $20 \mathrm{~S}$ proteasome, PA28 causes the $\mathrm{N}$-terminal tails of the $\alpha$-subunits to flip upwards, thereby facilitating substrate entry and product exit through the otherwise closed central 'gate' of the 20S proteasome [35, 36]. On the basis of these observations, it was suggested that such an open conformation might support the release of longer N-terminallyextended peptides, which were assumed to be adapted to the requirements of antigen presentation. However, binding of the 19S regulator also results in the complete opening of the gates [37]. Thus, it is unlikely that observed biological effects of PA28 function (see further down) are primarily the result of a gated $20 \mathrm{~S}$ proteasome configuration. Indeed, PA28-deficient mice are viable suggesting a more specialized function for this protein [38].

\section{Proteasomal antigen processing and contribution of immunosubunits}

The early finding that in vitro experiments, using purified $20 \mathrm{~S}$ proteasomes (standard and immuno), in combination with synthetic peptide substrates encompassing the epitopes and the natural flanking sequences, reproduced the observations made in intact cellular systems with surprisingly high fidelity [24, 39], led to a detailed analysis of a large number of different antigenic peptides of viral, bacterial and human origin. An important outcome of these experiments is that both standard and immunoproteasomes are able to generate MHC class I epitopes. Nevertheless, there can exist dramatic differences between the two subtypes with respect to the efficiency at which a given epitope can be generated. For example, experiments in which HeLa cells were infected with vaccinia virus expressing the hepatitis B virus (HBV) core antigen revealed an efficient liberation and presentation of the HBVcAg ${ }_{141-151}$ epitope [40]. However, presentation of this epitope essentially required the stimulation of $\mathrm{HeLa}$ cells with IFN- $\gamma$ and the formation of immunoproteasomes. Thus, no measurable peptide-specific CTL response was triggered in the presence of standard proteasomes only. Similarly, processing of a Mage-3-derived epitope essentially requires the presence of immunoproteasomes [41]. In contrast, the generation of a melanocyte Melan-A-derived tumor epitope was reported to be significantly impaired in the presence of immunoproteasomes [42].

Since the vast majority of peptides generated by the proteasome will be readily degraded by aminopeptidases [43, 44], the question arises how the sufficient and 
appropriate supply of MHC class I epitopes is guaranteed and whether immunoproteasomes and PA28 are involved in this process. Two alternative scenarios may apply. Immunoproteasomes/PA28 generate antigenic peptides using alternative cleavage sites. In this case, a qualitatively completely different set of peptides ought to be generated, which is possibly more adapted to TAP-dependent transport and the requirements of MHC class I binding than the one produced by standard proteasomes. Or, immunoproteasomes/PA28 generate a similar set of peptides as standard proteasomes, but by increased amounts and more efficiently to overcome the high peptide destruction rate within the cell to satisfy the needs of increased peptide supply. Approaching these questions from either a biochemical or more immunological perspective, however, will strongly influence the answers.

Experiments using a mouse embryonal cell line with TET-regulated expression of the immunosubunits and peptide-specific CTL assays as a 'read-out' system indicated that standard proteasomes barely generated the adenovirus E1B-derived epitope while the same epitope was efficiently generated by $20 \mathrm{~S}$ immunoproteasomes and that the amount of in vitro-generated E1B-derived closely correlated with the effect of immunoproteasomes in in vivo experiments [45]. Accepting CTL assays as the most sensitive immunological read-out to assess cellular proteasome function, one has to conclude that immunoproteasomes strongly determine the quality of epitope generation and as such also the immune recognition of a cell or tissue [46]. However, as determined by mass spectrometric (MS) analysis, standard proteasomes also generate "immunoproteasome-dependent" epitopes such as the adenovirus E1B epitope, albeit with a dramatically reduced efficiency. This finding was confirmed in later studies of E1B epitope presentation by GM-CSF-expanded dendritic cells (DC) that lacked immunosubunit expression ([47], and discussed below). Thus, the biochemical analysis reveals that the dramatic functional difference observed between the two proteasome subtypes by immune assays is not the consequence of the complete inability of standard proteasomes to generate the epitope or epitope precursor peptide but rather their relative inefficiency to do so. Combining this with other similar observations, it appears that immunoproteasomes strongly influence the available amount and therefore the quantity of a given epitope [48]. As a result, effects at the immunological level will become detectable only once a certain quantitative threshold of peptides is reached or failed to be reached. This does not exclude the possible existence of epitopes whose generation is indeed entirely dependent on one of two proteasome subtypes.

Mass spectrometric analysis of peptide fragments produced in vitro from different virus or tumor antigen-derived polypeptides harboring MHC class I epitopes indeed indicated that the presence of immunosubunits affects the cleavage site preference of the proteasome and in consequence will change the relative amounts at which an epitope or its precursor peptide is generated and hence will influence the immuno-dominance of epitopes [17]. A direct correlation of obtained mass signals with the amount of peptides generated is considerably complicated by the observation that, in many in vitro studies, substrate turnover by $20 \mathrm{~S}$ immunoproteasomes was found to be accelerated in comparison to standard proteasomes [49]. Therefore, in evaluating such studies and drawing conclusions for the in vivo situation, one has to discriminate between peptide fragments that are more abundant due to accelerated substrate turnover and those that are more prevalent due to altered proteasomal cleavage site usage. We recently solved this problem by introducing so-called antitopes, i.e. peptide fragments generated by cleavage within an epitope sequence but containing the correct $\mathrm{N}$-terminus of the epitope or the epitope precursor, as internal reference for the quantification of relative amounts of epitopes that are generated in vitro by either standard or immunoproteasomes [50]. Based on these analyses, it becomes evident that immunoproteasomes, when compared with standard proteasomes, exhibit an altered cleavage site preference with a strong predominance to cleave behind residues that represent the correct $\mathrm{C}$-terminus of a MHC class I epitope. Analysis of larger substrates also revealed that immunoproteasomes in many cases preferably generate epitope precursor peptides with a more extended N-terminal sequence that will facilitate TAP transport and require trimming by aminopeptidases [51]. Nevertheless, correct C-terminal cleavage site usage is not as robust as might be concluded from the biochemical data. Presumably, as part of an immune escape mechanism, mutations have naturally evolved affecting residues that directly flank the correct C-terminal cleavage site, resulting in strongly reduced epitope-generation efficiency. Thus, the recognition of the Moloney murine leukemia virus (MuLV)-derived epitope SSWDFTIV and the recognition of the p53-derived epitope spanning residues $264-272$ by specific CTLs was abolished due to a single amino acid substitution immediately flanking the C-terminal anchor residue of these epitopes [52]. An example of such an immune escape mutation is a so-called conservative $\mathrm{Y} / \mathrm{F}$ residue exchange flanking the immunodominant hepatitis $\mathrm{C}$ virus (HCV) NS3 ${ }_{1073-1081}$ epitope that was identified in a HCV strain isolated from chronically infected patients [53]. The $\mathrm{Y} / \mathrm{F}$ mutation impaired the proteasome's ability to generate the immunodominant NS3 ${ }_{1073-1081}$ epitope in any significant amount in vitro. Furthermore, infection of human leukocyte antigen (HLA)A2 transgenic mice with a vaccinia virus expressing the mutant NS3 protein demonstrated the inability of the proteasome to generate this epitope. These studies, therefore, 
are a direct experimental proof that in vitro processing reactions perfectly resemble the in vivo situation [53]. In addition, analysis of a CTL epitope encoded by AKV/MCF type of MuLV differing from the sequence in Friend/ Moloney/Rauscher (FMR) type in one residue within the epitope, as well as recent studies on a number of HIV epitopes, revealed that amino acid exchanges within an epitope sequence can also influence the proteasome's ability for correct or efficient C-terminal cleavage [54, 55]. Thus, although the preference of immunoproteasomes for cleavage behind residues that serve as anchors for MHC class I binding enhances antigen presentation, this immune mechanism is counteracted by viral adaptation to alter precisely these residues that allow efficient proteasomemediated epitope liberation.

\section{The role of PA28}

IFN- $\gamma$ modulates the activity of the cellular antigen-processing machinery not only through induction of immunoproteasome formation but also through the induced synthesis of the proteasome activator PA28 [56, 57]. PA28 binding to the $20 \mathrm{~S}$ proteasome strongly stimulates the hydrolysis of short fluorogenic peptide substrates, and detailed kinetic analysis showed that PA28 activates the 20S proteasome without affecting the active sites by either facilitating substrate entry or product exit [33]. Detailed biochemical studies, however, suggested that binding may effect substrate channelling and the accessibility of active sites for a given natural substrate, rather than affecting the characteristics of the active sites themselves [34].

The most striking evidence for structural changes imposed by PA28 on the 20S proteasomes was obtained when the processing and presentation of two CTL epitopes derived from a melanoma differentiation antigen, i.e., TRP2, was analyzed [58]. Detailed biochemical and molecular experiments showed that the inability of melanoma 18a cells to present the TRP $2_{360-368}$ epitope correlated with a strongly impaired expression of PA28 in these cells, which was also not restored by IFN- $\gamma$ treatment. Epitope presentation and the ability to activate TRP $2_{360-368}$ epitopespecific CTLs by melanoma 18a cells were, however, fully restored by transient transfection with cDNAs encoding the PA28 $\alpha$ and PA2 $\beta \beta$ subunits $[58,59]$. Interestingly, experiments using the PA28-independent TRP $2_{288-296}$ melanoma epitope and the murine cytomegalovirus-derived pp89 epitope precursor peptide for epitope replacement revealed that the flanking sequences of PA28-dependent TRP2 $2360-368$ epitope can transfer PA28 dependence onto otherwise PA28-independent epitopes. Moreover, the $\mathrm{N}$-terminal flanking sequence turned out to be sufficient to establish PA28 dependence of an epitope by allowing PA28-induced coordinated dual cleavages [60]. By which molecular interactions the $\mathrm{N}$-terminal flanking sequence promotes PA28 dependence remained unresolved in these studies, in particular since the flanking sequences of the human and mouse TRP2 epitope do not reveal any obvious similarities.

However, the TRP $2_{360-368}$ epitope generation is at present the only well-studied example of an absolute PA28 dependence and therefore it may rather represent the exception than the rule. Overexpression of PA28 in mouse fibroblasts was also shown to increase the sensitivity for lysis by CTLs directed against a MCMV-pp89-derived epitope [56]. In addition, TET-regulated expression of PA28 $\alpha \beta$ substantially increased the presentation of a MuLV gagL-derived CTL epitope [61]. In total, the investigation of several viral antigens shows that PA28 enhances the presentation of some viral epitopes without increasing overall protein turnover or the turnover of viral protein substrates, while the presentation of other virusderived epitopes was not affected [59]. Furthermore, this enhanced peptide presentation was independent of the presence of immunosubunits in the 20S proteasome [61] and, perhaps somewhat surprisingly, so far no cooperative effect between the two molecules has been observed [62].

Detailed in vitro analysis of the effects of PA28 on proteasomal cleavage site usage revealed that PA28, similar to immunoproteasomes, does not confer new cleavage specificities. Instead, PA28 markedly enhances the frequency of usage of specific preferred or minor cleavage sites, resulting in an immediate liberation of the intervening peptide fragments ([59], unpublished observation). Similar to immunoproteasomes, PA28 also induces a drastically accelerated turnover of synthetic polypeptide substrates that harbor MHC class I epitopes. Somewhat in contrast to immunoproteasomes, PA28 seems to affect the generation and presentation of only a selective and minor fraction of epitopes. Supporting this notion, it was shown that PA28 may also exert an allele-specific role by negatively influencing the processing of $\mathrm{K}^{\mathrm{d}}\left(\mathrm{D}^{\mathrm{d}}\right)$ ligands and by supporting the generation of $\mathrm{K}^{\mathrm{b}}\left(\mathrm{L}^{\mathrm{d}}\right)$ epitopes [63]. Thus, PA28 seems not to be a prerequisite for antigen presentation in general, playing a role for the processing of only certain epitopes. Considering that, independent of cytokine induction, PA28 is expressed in almost all tissues or cells analyzed, one is led to infer that the observed involvement of PA28 in antigen presentation may be only a secondary effect of a not yet resolved true physiological function of PA28.

\section{Expression patterns of proteasome immunosubunits in vivo}

As mentioned above, analyses of both cell lines and different mouse tissues have demonstrated that the 
immunosubunits, as well as in IFN $\gamma$-stimulated cells, are expressed mainly in the lymphoid tissues such as thymus, spleen and lymph nodes [64]. In lymphoid tissues, especially immature, DC express the immunosubunits at high levels, which are only slightly upregulated following maturation, or even downregulated, depending on the maturation-inducing stimuli received [65-68]. Immunosubunit expression was further demonstrated for macrophages [69], T cells [68] and B cells [70, 71], where immunoproteasome abundance varied with the state of differentiation [72]. Compared to lymphoid tissues, the abundance of the immunosubunits in non-lymphoid peripheral tissues is rather low [64, 73]. The residual levels of immunosubunits, as detected by analysis of the tissues ex vivo, in part may be explained by the presence of, for example, DC and macrophages. However, most likely immunosubunit expression in these tissues is not entirely restricted to cells of hematopoietic origin. For example, primary hepatocytes have been shown to express low amounts of, mainly, LMP7, also in the absence of cytokine stimulation [74, 75].

\section{In vivo formation of immunoproteasomes during infection}

Because stimulation of cells that were maintained in tissue culture with IFN $\gamma$, as well as with $\operatorname{IFN} \alpha, \beta$ or TNF $\alpha[74,76$, 77], upregulated immunosubunit expression, the question arose whether (pro)inflammatory cytokines, released over the course of pathogen-induced immune responses, altered the composition of proteasomes in vivo. Indeed, both infections with LCMV and with Listeria monocytogenes were found to lead to an exchange of constitutive for immunoproteasomes in livers of infected mice [48, 78]. Analysis of infected, IFN $\gamma$, and IFN- $\alpha$, receptor gene-deficient mice showed that, in the LCMV model, approximately $50 \%$ of the replacement of constitutive by immunoproteasomes was dependent on IFN $\gamma$, whereas type 1 IFNs played a minor role [78]. In the case of Listeriainfected mice, proteasomes of different tissues were purified and tested for their ability to generate an immunoproteasome-dependent Listeria epitope. In tissues lacking the IFN $\gamma$ R, but not the TNFR, the production of this epitope was not upregulated following infection, indicating an important role for IFN $\gamma$ in regulation of epitope generation following infection with this bacterium [48]. Also, infection of mice with Histoplasma capsulatum, a fungus that induces strong Th1-biased immune responses, led to replacement of constitutive for immunoproteasomes in the infected tissues [75], which in this model was strictly dependent on IFN $\gamma$. In contrast, in chimpanzees infected with $\mathrm{HCV}$ infection, increases in immunosubunit expression in inflamed liver occur well before upregulation of IFN $\gamma$ (and TNF $\alpha$ ) expression, and coincide with type I IFN responses [74]. Since exposure to type I IFN enhances the expression of these proteasome components in a hepatocyte cell line and primary hepatocytes in cell culture, these data suggest that type I IFNs induce the upregulation of immunoproteasome formation during acute $\mathrm{HCV}$ infection. Taken together, these studies indicate that both proinflammatory cytokines, which are already released by innate immune cells in a very early stage of infection, as well as IFN $\gamma$ which is released by NK cells, and T cells that arrive approximately one to several days later at the site of infection, contribute to the replacement of constitutive by immunoproteasomes in inflamed tissues.

\section{Effects of immunoproteasome expression on pathogen-specific $\mathrm{CD8} \mathrm{T}$ cell responses}

Pathogen-specific CD8 T cell responses are usually directed to only one immunodominant and a few subdominant epitopes. The observation that DC express the IFN $\gamma$ inducible components permanently, whereas in peripheral tissues the expression of these proteasome components as well as that of other components of the MHC class I antigen processing pathway is induced during infection, both by proinflammatory cytokines and IFN $\gamma$, strongly suggests that immunoproteasomes serve to produce the epitopes, detected by the pathogen-specific CD8 $\mathrm{T}$ cell response. Thus, immediately after infection, pathogenderived antigens are processed by immunoproteasomecontaining DC and presented to naïve CD8 T cells, which then are activated and start proliferating. Approximately 4 days later, fully functional, antigen-specific CD8 T cells will migrate to the inflamed tissue, and there recognize the epitopes, generated by the induced immunoproteasomes. Indeed, different studies have demonstrated that immunoproteasome-mediated antigen processing shapes the finespecificity of pathogen-specific CD8 T cell responses. For example, quantifying CD8 $\mathrm{T}$ cell responses to seven viral epitopes during influenza virus (PR8) infection in mice, Chen et al. [79] found that the magnitude of the responses to two dominant epitopes were dramatically decreased in LMP2-gene-deficient compared to $w t$ mice, whereas the frequencies of CD8 $\mathrm{T}$ cells responding to two subdominant epitopes were increased. Further analyses of antigen presentation kinetics by infected LMP2-deficient compared to $w t$ cells in vitro, and of expansion of adoptively transferred wt CD8 $\mathrm{T}$ cells in PR8-infected LMP2-deficient mice, showed that quantitative differences in epitope presentation explained the decreased response to one of the dominant epitopes, derived from the viral acid polymerase (PA), in LMP2-deficient mice, as well as the enhanced 
response to one of the subdominant epitopes. These experiments, however, also indicated that the poor CD8 T cell responses to the second, dominant influenza epitope, in the absence of LMP2, was not explained by aberrations in viral epitope generation but probably by deficiencies in $T$ cell repertoire. Thus, in addition to processing of pathogenderived antigens, the immunosubunits may also influence $\mathrm{T}$ cell selection, as will be discussed below. Further studies by Pang et al. [80] showed that, like LMP2-deficient mice, LMP7 + MECL-1-deficient mice also failed to process and present the influenza PA-derived epitope efficiently, and mounted very weak CD8 T cell responses to this epitope following infection with PR8. Importantly, insertion of the PA epitope sequences in the neuraminidase stalk restored proteasome-mediated generation of this eptitope in immunosubunit-deficient cells, and thereby rescued the ability of the epitope to elicit dominant CD8 T cell responses in immunosubunit-deficient mice, infected with the mutant influenza strain. Also, in the murine model of LCMV infection, proteasome composition was found to determine the ability of infected cells to process and present different LCMV-derived epitopes [69]. Enhanced presentation of one epitope, in the absence of LMP2 or LMP7, correlated with higher frequencies of CD8 T cells responding to this epitope in LCMV-infected LMP2 or LMP7-gene-deficient compared to $w t$ mice. Thus, in this example, immunoproteasomes limit efficient generation of one of the viral epitopes, and thereby the size of CD8 T cell responses triggered by this epitope. A shift in immunodominance hierarchy correlating with altered antigen processing was also observed in immunosubunit-deficient HLA-A2/Kb transgenic mice that received a DNA prime, recombinant vaccinia boost vaccination with the HBV env and pol proteins [81]. In the absence of LMP7, numbers of CD8 $\mathrm{T}$ cells reacting to two epitopes were decreased. Responses to a third epitope were increased compared to vaccinated control mice.

To determine how altered efficiencies of epitope liberation influence the magnitude of CD8 T cell responses, a recombinant $L$. monocytogenes strain (rLM-E1) secreting a hybrid protein that encompassed the immunoproteasomedependent adenovirus E1B epitope was constructed [47]. Analysis of rLM-E1-infected BM-DC with peptide-specific CTL revealed that deficiency of LMP7 and MECL-1 delayed (but did not abolish) the presentation of the E1B epitope. Consistent with this defect in antigen processing, LMP7 + MECL-1-deficient mice failed to mount E1Bspecific CD8 $\mathrm{T}$ cell responses following infection with rLM-E1. In contrast, E1B was the dominant target of the rLM-E1-specific CD8 $\mathrm{T}$ cell response in infected $w t$ controls. Alterations in TCR repertoire could not explain the unresponsiveness of LMP7 + MECL-1-deficient mice, as immunization with DC loaded with synthetic E1B peptide primed E1B-specific CD8 T cells with high TCR avidity. These $\mathrm{T}$ cells formed memory and expanded following secondary infection with rLM-E1, indicating that the E1B epitope was presented in rLM-E1-infected immunosubunit-deficient mice and triggered the re-activation of CD8 T cell memory, but could not prime naïve CD8 T cells during primary infection. Remarkably, LMP7 + MECL-1deficient mice also raised E1B-specific CD8 T cell responses after receiving splenic APC of LMP7 + MECL-1-deficient mice, infected $36 \mathrm{~h}$ earlier with rLM-E1. Thus, after $36 \mathrm{~h}$, the quantities of E1B on the APC were sufficient to elicit a CD8 T cell response. Taken together, these findings indicate that the priming of $\mathrm{CD} 8 \mathrm{~T}$ cells requires that their ligands reach a specific threshold level on the priming pAPC, already at an early time point following infection. We conclude that immunoproteasomes, next to other contributing factors, can serve to lift the cell surface density of specific epitopes over this threshold.

In conclusion, the changes in proteasome cleavage preferences induced by immunosubunit incorporation affect the efficiency of generation of multiple pathogenderived epitopes, and thereby the fine specificity of pathogen-specific CD8 T cell responses. Nevertheless, there is no compelling evidence that immunoproteasome-mediated antigen processing plays a major role in pathogen resistance. As reviewed above, the different immunosubunitdeficient mice analyzed so far mount pathogen-specific CD8 $\mathrm{T}$ cell responses, although with different immunodominance hierarchies, and acute infections with pathogens such as LCMV are cleared with similar kinetics in LMP2and LMP7-deficient mice as in $w t$ mice [82]. On the other hand, in a mouse model of CVB3 infection, Jäkel et al. [83] observed that early type I interferon responses and concomitant upregulation of immunosubunit expression, along with other components of the MHC class I antigen processing pathway, correlated with resistance to chronic infection. LMP7-deficient mice show an enhanced suscepitibility to Toxoplasma infection [84] and, remarkably, a delayed clearance of $L$. monocytogenes from the liver that correlated with diminished production of an immunoproteasome-dependent epitope [48]. Thus, analyses of a large variety of infection models including different types of pathogens may lead to a readjustment of our present view, i.e. that immunoproteasomes play a limited role in pathogen resistance.

\section{A new pool of proteasome-generated antigenic peptides}

Newly translated polypeptides, a large pool of which was ubiquitylated, are thought to represent an important, yet not the sole, source for MHC class I epitopes [5, 85]. It was thus proposed, though not formally shown, that this pool is 
mostly composed of defective proteins, hence called defective ribosomal products (DRiPs). The DRiP hypothesis, although not undisputed, not only links antigen processing to translation but would also guarantee that the cellular immune system has access to its substrates before they reach their final destination within or outside the cell [5, 85-87].

Until recently, there existed the canonical rule that antigenic peptides of 8-10 residues in length, or precursor peptides thereof which are generated by the $20 \mathrm{~S}$ proteasome, are peptide fragments with a linear sequence identical to that found in the unprocessed parental protein.

This view, however, was dramatically changed by reports in which antigenic peptides were identified which were composed of two different peptide fragments whose sequence was not contiguous in the parent protein. These epitope peptides, identified by patient derived CTL were post-proteolytic fusions of proteasomal cleavage products and were shown to be generated by proteasome catalyzed peptide splicing (PCPS) in a proposed transpeptidation reaction (see also the review by Vigneron et al. [88]).

The first spliced epitope peptide identified [89] representing tumor epitope was derived from FGF-5 (fibroblast growth factor) and was encoded as such by the FGF-5 gene but it was a fusion of a five-residue peptide with a fourresidue peptide that was located 40 residues away. Although the underlying mechanism was not elucidated at the time, it appeared likely that the spliced peptide was generated by the proteasome.

Consequently, two additional spliced immunogenic peptides, derived from the melanocyte protein gp100 and the SP100 nuclear phosphoprotein that were recognized by CTLs, were identified [90]. Biochemical analysis convincingly showed that splicing of the antigenic peptides is catalyzed by the $20 \mathrm{~S}$ proteasome in a reverse proteolysis mechanism. In short, cleavage by the proteasome is the result of a nucleophilic attack on the peptide bond by the catalytic threonines that exist on the N-terminus of the $\beta 1$, $\beta 2$ and $\beta 5$ subunits or their IFN- $\gamma$ induced homologues, $\beta 1 \mathrm{i}, \beta 2 \mathrm{i}$ and $\beta 5 \mathrm{i}$, in the immunoproteasome. The attack results in the formation of an acylester intermediate. Usually, these peptides are released from the proteasomes by rapid hydrolyzation. Only when the acylester is stabilized at the active site for a sufficient time span can $\mathrm{N}$-termini of released peptide fragments compete with water molecules and make a nucleophilic attack on the ester bond of the acyl-enzyme intermediate, forming a new peptide bond producing the spliced peptide. Furthermore, it was also shown by Warren et al. [91] that two non-contiguous peptides can also be fused by splicing in a reversed order confirming that the splicing reaction is dependent on the retention time of the acyl-intermediate at the active site threonine. The reversed splicing reaction also showed that, after formation of the acyl-ester, the peptide is released into the catalytic cavity of the proteasome where it can compete with other peptides for the splicing reaction. This means that, in theory, and neglecting the so far unknown sequence requirements, any peptide fragment generated by proteasomal hydrolysis can participate in a splicing reaction thereby giving rise to an unforeseen number of potential splicing products and putative epitope containing peptides.

Since, under physiological conditions proteolysis is normally favored over hydrolysis, the formation of new immunologically relevant MHC class I ligands by PCPS raised the possibility that reverse proteolysis may be functionally more important than previously thought. On the other hand, one has to acknowledge that only three spliced epitope peptides have been reported in the literature since their initial discovery in 2004 [89-92]. Thus, in accord with the biochemical rules, PCPS is discussed to be a relatively rare event and therefore supposed to be of very limited immunological relevance. However, since not every spliced peptide will fulfil the requirement of a MHC class I ligand, the peptide splicing reaction as such may be a considerably more frequent side reaction of proteasome hydrolysis than previously assumed. But even if peptide splicing is a relative rare event, PCPS may still play an important role within the immune response. This is due to the sensitivity of $\mathrm{CD} 8+\mathrm{T}$ cells, which are able to detect very small numbers of MHC class I peptide complexes, and in the most extreme example even a single MHC class I complex.

However, so far, all presently available database search algorithms fail to detect spliced peptide products. We recently developed a theoretical/experimental method, named SpliceMet, which combines the computer-based algorithm ProteaJ with MS analyses of proteasome-generated peptides which in future will facilitate a systematic and widely CTL-independent identification of spliced peptides.

\section{Role of proteasome subunits in selection of the TCR repertoire}

$\mathrm{T}$ cells develop in the thymus and there are positively selected for ability to recognize self-MHC, and then negatively selected to eliminate $\mathrm{T}$ cells bearing TCR that recognize autoantigens. While positive selection is based on weak interactions of TCR with MHC molecules presenting peptides of cellular proteins and stimulates survival, negative selection is based on strong interactions with peptide-loaded MHC molecules and induces apoptosis. Surprisingly, both Chen et al. [79] and Basler et al. [93], analyzing CD8 $\mathrm{T}$ cell responses to influenza virus in LMP2-deficient and LCMV in MECL-1-deficient mice 
(see above), found that specific epitopes that were processed and presented efficiently failed to trigger $\mathrm{T}$ cell responses in the absence of LMP2 or MECL-1. Indeed, $w t$ CD8 T cells transferred into LMP2- or MECL-1-deficient mice responded to these epitopes following infection with influenza or LCMV. These findings led to the infererence that the decreased responses in LMP2- and MECL-1-deficient mice were explained by alterations in the TCR repertoire, and thus also strongly suggested an important role for immunoproteasomes in peptide presentation in the thymus and shaping of the TCR repertoire.

Further insight into the role of proteasome cleavage preferences in CD8 $\mathrm{T}$ cell selection relies on recent data by Murata et al. [73], who discovered a novel proteasome subunit, $\beta 5 \mathrm{t}$. The gene encoding $\beta 5 \mathrm{t}$ was identified in a search for proteasome-related genes in a genome database, and appeared to be located adjacent to the gene encoding $\beta 5$. $\beta 5 \mathrm{t}$ was found to be expressed only in cortical thymical epithelial cells (cTEC), and to incorporate preferentially into proteasome precursor complexes containing LMP2 and MECL-1 [73]. A comparison of peptidase activities of $20 \mathrm{~S}$ and $26 \mathrm{~S}$ containing $\beta 5$ or $\beta 5 \mathrm{t}$ showed that $\beta 5 \mathrm{t}$ incorporation selectively reduced the proteasomal chymotrypsin-like activity. Thus, these findings suggest that $\beta 5 \mathrm{t}$ incorporation alters the proteasomal cleavage site usage in protein substrates, and thereby the repertoire of peptides available for MHC class I binding in cells expressing this subunit. In concordance with this conclusion, $\beta 5$ t-deficient mice showed a severe reduction in numbers of single positive CD8 $\mathrm{T}$ cells in the thymus and periphery, to approximately 20 and $30 \%$, respectively, of CD8 T cells in control mice, and failed to positively select transgenic TCR recognizing the HY-antigen, an LCMVand an influenza-derived CD8 T cell epitope [73, 94]. The severe impact of $\beta 5 \mathrm{t}$ on CD8 T cell repertoire was further illustrated by the finding that mice lacking this subunit showed a markedly enhanced susceptibility to influenza virus infection [94], during which CD8 T cells are critical for viral clearance [95]. On the other hand, ova-specific T cells bearing the OT- $1 \mathrm{tg}$ TCR were selected in $\beta 5$ t-deficient mice [94], and these cells were responsive to restimulation with ova peptide, ex vivo, indicating the remaining CD8 $\mathrm{T}$ cells are functional. Taken together, these data indicate that the cTEC-expressed $\beta 5 \mathrm{t}$ subunit plays an important role in positive selection of CD8 T cells in the thymus. $\beta 5 \mathrm{t}$ is found in $20 \mathrm{~S}$ complexes mainly in conjunction with LMP2 and MECL-1. This finding nicely fits with the observations reviewed above [79, 93] that these subunits influence $\mathrm{T}$ cell selection, although to a much lesser extent than $\beta 5 \mathrm{t}$. Conversely, LMP7 and not $\beta 5 \mathrm{t}$ is critically involved in selection of the OT-1 TCR, shown previously by Osterloh et al. [96], using LMP7-deficient mice and confirmed by the more recent observations in
B5t-deficient mice, discussed above [94]. In support of a role of LMP7 in positive selection, two-dimensional PAGE of 20S complexes of cTECs, immunoprecipitated with anti- $\beta 5 \mathrm{t}$ antibody, showed a faint spot for LMP7, indicating that a fraction of the $\beta 5 \mathrm{t}$-containing proteasomes in cTEC are mixed complexes, also containing LMP7 [73]. Taken together, these data indicate a predominant role for the cTEC-expressed $\beta 5 \mathrm{t}$ subunit in the production of MHC class I-presented peptides that mediate positive selection and minor roles for LMP2, MECL-1 and LMP7.

\section{Evidence for non-antigen processing-related roles of immunoproteasomes in immune responses}

The main function of proteasomes is the degradation of intracellular proteins that are either defective or tagged, usually by polyubquitin moieties. By selective protein degradation, proteasomes regulate many cellular processes including antigen processing, but also the activation of transcription factors, cell proliferation and differentiation. Thus, although most studies had focused on the role of immunoproteasomes in antigen processing and CD8 T cellmediated immune responses, it remained plausible that the immunosubunits could play a more pleiotrope role. Moreover, mainly lymphoid cells express the immunosubunits in an IFN $\gamma$-independent fashion, suggesting that these additional functions of immunoproteasomes may impact on immune responses. First observations in support of this possibility were by Chen et al. [79], who noticed that the overall magnitude of the $\mathrm{CD} 8 \mathrm{~T}$ cell response to influenza virus in LMP2-deficient mice was significantly lower than in $w t$ mice. In addition, LMP2-deficient CD8 T cells transferred into $w t$ mice failed to expand following influenza infection, as was also later found for $\mathrm{T}$ cells lacking MECL-1 [93] or MECL-1 and LMP7 [79, 80]. Thus, the immunosubunits may be involved in lymphocyte survival or expansion. Recently, Hensley et al. [70] reported that LMP2-deficient but not LMP2 + MECL-1or LMP7 + MECL-1-deficient mice displayed severely reduced numbers of mature B cells, CD4 and CD8 T cells in the spleen. Further analyses of the LMP2-deficient mouse strain revealed severe defects in B cell and DC function, resulting in reduced primary anti-viral antibody responses with reduced isotype switch, and a reduced production of different pro-inflammatory cytokines by influenza virus-infected LMP2-deficient BM DC in cell culture. LPS-stimulated LMP2-deficient B cells showed reduced $\mathrm{i} \kappa \mathrm{B}$ degradation, suggesting that deficient $\mathrm{NF} \kappa \mathrm{B}$ activation may explain some of the defects observed in LMP2-deficient mice. Because mice lacking both LMP2 and MECL-1 did not show any major aberrations in 
cellular subsets in the spleen, the defects of mice lacking LMP2 were attributed to the formation of mixed proteasomes, containing $\beta 1$ in combination with MECL- 1 and LMP7, a proteasome type that is disfavored when LMP2 is present.

Different studies in mice lacking MECL-1 or both LMP7 and MECL-1 further supported a role for immunosububunits in $\mathrm{T}$ cell proliferation. Caudill et al. [97] reported that the absence of MECL-1 or of both LMP7 and MECL-1 led to enhanced CD4/CD8 ratios in the peripheral lymphoid tissues. A further analysis of CD4/CD8 $\mathrm{T}$ cell ratios in $\mathrm{BM}$ chimeric mice showed that the ratios of LMP7 + MECL-1-deficient CD4/CD8 T cells were also enhanced when these $\mathrm{T}$ cells developed in $w t$ mice [98]. Strikingly, in mixed chimeric mice, consisting of an LMP7 + MECL-1-deficient recipient reconstituted with a mix of LMP7 + MECL-1-deficient and wt BM, the immunosubunit-deficient and $w t \mathrm{~T}$ cell populations maintained their characteristically different CD4/CD8 ratios, despite the fact that these $\mathrm{T}$ cells developed in the same thymic environment and circulated in the same peripheral lymphoid tissues [98]. Thus, as environmental factors cannot explain the aberrant relative expansion of the CD4 and CD8 $\mathrm{T}$ cell subsets in mice lacking MECL-1, it must be concluded that $\mathrm{T}$ cell-expressed MECL-1 regulates homeostatic $\mathrm{T}$ cell expansion or survival, perhaps by altered degradation of anti- or pro-apoptotic proteins or altered transcription factor activation.

A recent study [99] demonstrated that treatment with an LMP7-selective inhibitor, PR-957, reduced the production of TNF $\alpha$, IL-23 and IL-6 by LPS-stimulated human PBMC and of IFN $\gamma$ and IL-2 by activated human T cells. Also, activated $w t$ but not LMP7-deficient mouse splenocytes produced reduced amounts of cytokines following PR-957 treatment, confirming that the observed effects were a result of LMP7-inhibition. Remarkably, no differences in cytokine production were observed between untreated $w t$ and LMP7-deficient mouse splenocytes, demonstrating that the constitutive subunits/ $\beta 5$ can compensate for the role of LMP7 in cytokine production. To determine whether selective inhibition of LMP7 impacts on ongoing inflammatory responses in vivo, mice with collagen- or collagen antibody-induced arthritis were treated with PR-957. In both models, experimental arthritis was ameliorated following injection of PR-957, probably due to reduced production of inflammatory cytokines and reduced cellular infiltration. Taken together, these data as well as the findings by Hensley at al. in LMP2-deficient mice ([70]; see above) indicate that proteasomes play an important role in regulation of cytokine production, and thereby reveal yet another important function of (immuno) proteasomes.

\section{Defective ribosomal products, immunoproteasomes and peptide quantity}

Poly-ubiquitylated nascent defective proteins (DRiPs) are thought to represent the major but not the sole source for MHC class I peptide ligands [5, 85] (for details, see the review by Dolan et al. [100]). The DRiP hypothesis, which links antigen processing to translation, would guarantee that the cellular immune system has immediate access to its substrates, and thus uncouples antigen presentation from intracellular protein destination or half-life [5, 85-87]. However, the idea that a large amount of newly translated proteins should be misfolded or harbor translational errors also raised some doubts with regard to the validity of the hypothesis [101, 102]. Thus, considering that all major components of the antigen processing pathway are upregulated upon IFN stimulation and that the definition of "defect" remained unclear, there also existed no explanation of how DRiP levels can be adapted to changing immunological requirements in terms of peptide supply.

Recently, a somewhat surprising solution to this problem has been offered by Seifert and colleagues [103]. Their study revealed that IFNs trigger a strong and transient accumulation of oxidant-damaged nascent poly-ubiquitylated proteins and that, under proinflammatory conditions, DRiPs from a pool of oxidized, unfolded nascent proteins marked by poly-ubiquitin chains underwent rapid degradation by the $26 \mathrm{~S}$ proteasome. The increase in the DRiPs pool is brought about by the IFN-induced formation of ROS, mTOR-dependent enhanced translation and strongly increased poly-ubiquitylation activity. Interestingly, during the early phase of IFN stimulation, the generation of these DRiPs significantly exceeds the degradation capacity of the UPS leading to a strong, transient accumulation of polyubiquitylated proteins and formation of aggresome-likeinduced structures (ALIS). The elimination and efficient turnover of these accumulated defective and ubiquitylated proteins essentially requires the presence of $26 \mathrm{~S}$ immunoproteasomes, exhibiting an approximately three- to fourfold higher degradation capacity than standard $26 \mathrm{~S}$ proteasomes. Supporting their importance in overcoming IFN-induced oxidative stress, the absence of immunoproteasomes, as in LMP7-deficient mice, resulted in the inability of cells to eliminate the accumulating DRiPs, in enhanced ALIS formation and increased susceptibility to apoptosis. These experiments show that physiologically a primary function of immunopoteasomes resides in the maintenance of protein homeostasis under proinflammatory conditions and that, in consequence, as a result of its enhanced degradation capacity, immuno-26S proteasomes also strongly expand the pool of peptides available for MHC class I antigen presentation. 
In conclusion, the proteasome system plays a fundamental role in immune regulation through a variety of mechanisms including MHC class I antigen processing, lymphocyte survival and regulation of cytokine production/ inflammation. Consequently, inhibition of specific proteasomal active sites may impact on all these proteasomemediated processes, which should be carefully considered when analyzing the role of specific proteasome components in specific immune effector functions.

Open Access This article is distributed under the terms of the Creative Commons Attribution Noncommercial License which permits any noncommercial use, distribution, and reproduction in any medium, provided the original author(s) and source are credited.

\section{References}

1. Rock KL, Goldberg AL (1999) Degradation of cell proteins and the generation of MHC class I-presented peptides. Annu Rev Immunol 17:739-779

2. Falk K, Rotzschke O, Deres K et al (1991) Identification of naturally processed viral nonapeptides allows their quantification in infected cells and suggests an allele-specific $\mathrm{T}$ cell epitope forecast. J Exp Med 174(2):425-434

3. Rammensee HG, Friede T, Stevanoviic S (1995) MHC ligands and peptide motifs: first listing. Immunogenetics 41(4):178-228

4. Rock KL, Gramm C, Rothstein L et al (1994) Inhibitors of the proteasome block the degradation of most cell proteins and the generation of peptides presented on MHC class I molecules. Cell 78(5):761-771

5. Reits EA, Vos JC, Gromme M, Neefjes J (2000) The major substrates for TAP in vivo are derived from newly synthesized proteins. Nature 404(6779):774-778

6. Momburg F, Hammerling GJ (1998) Generation and TAPmediated transport of peptides for major histocompatibility complex class I molecules. Adv Immunol 68:191-256

7. Kloetzel PM (2001) Antigen processing by the proteasome. Nat Rev Mol Cell Biol 2(3):179-187

8. Ciechanover A (2005) Proteolysis: from the lysosome to ubiquitin and the proteasome. Nat Rev Mol Cell Biol 6(1):79-87

9. Glickman MH, Rubin DM, Coux O et al (1998) A subcomplex of the proteasome regulatory particle required for ubiquitinconjugate degradation and related to the $\mathrm{COP} 9$-signalosome and eIF3. Cell 94(5):615-623

10. Voges D, Zwickl P, Baumeister W (1999) The 26S proteasome: a molecular machine designed for controlled proteolysis. Annu Rev Biochem 68:1015-1068

11. Groll M, Ditzel L, Lowe J et al (1997) Structure of $20 \mathrm{~S}$ proteasome from yeast at 2.4 A resolution. Nature 386(6624): 463-471

12. Groll M, Bajorek M, Kohler A et al (2000) A gated channel into the proteasome core particle. Nat Struct Biol 7(11):1062-1067

13. Schmidtke G, Eggers M, Ruppert $T$ et al (1998) Inactivation of a defined active site in the mouse $20 \mathrm{~S}$ proteasome complex enhances major histocompatibility complex class I antigen presentation of a murine cytomegalovirus protein. J Exp Med 187(10):1641-1646

14. Nussbaum AK, Dick TP, Keilholz W et al (1998) Cleavage motifs of the yeast $20 \mathrm{~S}$ proteasome beta subunits deduced from digests of enolase 1. Proc Natl Acad Sci USA 95(21):12504 12509
15. Kisselev AF, Akopian TN, Woo KM, Goldberg AL (1999) The sizes of peptides generated from protein by mammalian 26 and $20 \mathrm{~S}$ proteasomes. Implications for understanding the degradative mechanism and antigen presentation. J Biol Chem 274(6): 3363-3371

16. Schwarz K, de Giuli R, Schmidtke G et al (2000) The selective proteasome inhibitors lactacystin and epoxomicin can be used to either up- or down-regulate antigen presentation at nontoxic doses. J Immunol 164(12):6147-6157

17. Groettrup M, Soza A, Kuckelkorn U, Kloetzel PM (1996) Peptide antigen production by the proteasome: complexity provides efficiency. Immunol Today 17(9):429-435

18. Glynne R, Powis SH, Beck S et al (1991) A proteasome-related gene between the two $\mathrm{ABC}$ transporter loci in the class II region of the human MHC. Nature 353(6342):357-360

19. Brown MG, Driscoll J, Monaco JJ (1991) Structural and serological similarity of MHC-linked LMP and proteasome (multicatalytic proteinase) complexes. Nature 353(6342): 355-357

20. Kelly A, Powis SH, Glynne R et al (1991) Second proteasomerelated gene in the human MHC class II region. Nature 353(6345):667-668

21. Ortiz-Navarrete V, Seelig A, Gernold M et al (1991) Subunit of the '20S' proteasome (multicatalytic proteinase) encoded by the major histocompatibility complex. Nature 353(6345):662-664

22. Aki M, Shimbara N, Takashina M et al (1994) Interferongamma induces different subunit organizations and functional diversity of proteasomes. J Biochem 115(2):257-269

23. Driscoll J, Brown MG, Finley D, Monaco JJ (1993) MHC-linked LMP gene products specifically alter peptidase activities of the proteasome. Nature 365(6443):262-264

24. Boes B, Hengel H, Ruppert T et al (1994) Interferon gamma stimulation modulates the proteolytic activity and cleavage site preference of 20S mouse proteasomes. J Exp Med 179(3):901909

25. Kuckelkorn U, Frentzel S, Kraft R et al (1995) Incorporation of major histocompatibility complex-encoded subunits LMP2 and LMP7 changes the quality of the $20 \mathrm{~S}$ proteasome polypeptide processing products independent of interferon-gamma. Eur $\mathrm{J}$ Immunol 25(9):2605-2611

26. Ustrell V, Pratt G, Rechsteiner M (1995) Effects of interferon gamma and major histocompatibility complex-encoded subunits on peptidase activities of human multicatalytic proteases. Proc Natl Acad Sci USA 92(2):584-588

27. Heink S, Ludwig D, Kloetzel PM, Kruger E (2005) IFN-gammainduced immune adaptation of the proteasome system is an accelerated and transient response. Proc Natl Acad Sci USA 102(26):9241-9246

28. Dubiel W, Pratt G, Ferrell K, Rechsteiner M (1992) Purification of an $11 \mathrm{~S}$ regulator of the multicatalytic protease. J Biol Chem 267(31):22369-22377

29. Ma CP, Slaughter CA, DeMartino GN (1992) Identification, purification, and characterization of a protein activator (PA28) of the 20S proteasome (macropain). J Biol Chem 267(15): $10515-10523$

30. Hendil KB, Khan S, Tanaka K (1998) Simultaneous binding of PA28 and PA700 activators to $20 \mathrm{~S}$ proteasomes. Biochem $\mathrm{J}$ 332(Pt 3):749-754

31. Tanahashi N, Murakami Y, Minami Y et al (2000) Hybrid proteasomes. Induction by interferon-gamma and contribution to ATP-dependent proteolysis. J Biol Chem 275(19):1433614345

32. Realini C, Dubiel W, Pratt G et al (1994) Molecular cloning and expression of a gamma-interferon-inducible activator of the multicatalytic protease. J Biol Chem 269(32):20727-20732 
33. Stohwasser R, Salzmann U, Giesebrecht J et al (2000) Kinetic evidences for facilitation of peptide channelling by the proteasome activator PA28. Eur J Biochem 267(20):6221-6230

34. Li J, Gao X, Joss L, Rechsteiner M (2000) The proteasome activator 11S REG or PA28: chimeras implicate carboxyl-terminal sequences in oligomerization and proteasome binding but not in the activation of specific proteasome catalytic subunits. J Mol Biol 299(3):641-654

35. Knowlton JR, Johnston SC, Whitby FG et al (1997) Structure of the proteasome activator REGalpha (PA28alpha). Nature 390(6660):639-643

36. Whitby FG, Masters EI, Kramer L et al (2000) Structural basis for the activation of $20 \mathrm{~S}$ proteasomes by $11 \mathrm{~S}$ regulators. Nature 408(6808): $115-120$

37. Gillette TG, Kumar B, Thompson D et al (2008) Differential roles of the COOH termini of AAA subunits of PA700 (19 S regulator) in asymmetric assembly and activation of the $26 \mathrm{~S}$ proteasome. J Biol Chem 283(46):31813-31822

38. Murata S, Udono H, Tanahashi N et al (2001) Immunoproteasome assembly and antigen presentation in mice lacking both PA28alpha and PA28beta. EMBO J 20(21):5898-5907

39. Niedermann G, King G, Butz S et al (1996) The proteolytic fragments generated by vertebrate proteasomes: structural relationships to major histocompatibility complex class I binding peptides. Proc Natl Acad Sci USA 93(16):8572-8577

40. Sijts AJ, Ruppert T, Rehermann B et al (2000) Efficient generation of a hepatitis $\mathrm{B}$ virus cytotoxic $\mathrm{T}$ lymphocyte epitope requires the structural features of immunoproteasomes. J Exp Med 191(3):503-514

41. Schultz ES, Chapiro J, Lurquin C et al (2002) The production of a new MAGE-3 peptide presented to cytolytic T lymphocytes by HLA-B40 requires the immunoproteasome. J Exp Med 195(4): 391-399

42. Morel S, Levy F, Burlet-Schiltz O et al (2000) Processing of some antigens by the standard proteasome but not by the immunoproteasome results in poor presentation by dendritic cells. Immunity 12(1):107-117

43. Princiotta MF, Schubert U, Chen W et al (2001) Cells adapted to the proteasome inhibitor 4-hydroxy-5-iodo-3-nitrophenylacetylLeu-Leu-leucinal-vinyl sulfone require enzymatically active proteasomes for continued survival. Proc Natl Acad Sci USA 98(2):513-518

44. Reits E, Neijssen J, Herberts C et al (2004) A major role for TPPII in trimming proteasomal degradation products for MHC class I antigen presentation. Immunity 20(4):495-506

45. Sijts AJ, Standera S, Toes RE et al (2000) MHC class I antigen processing of an adenovirus CTL epitope is linked to the levels of immunoproteasomes in infected cells. J Immunol 164(9): 4500-4506

46. Toes RE, Nussbaum AK, Degermann S et al (2001) Discrete cleavage motifs of constitutive and immunoproteasomes revealed by quantitative analysis of cleavage products. J Exp Med 194(1):1-12

47. Deol P, Zaiss DM, Monaco JJ, Sijts AJ (2007) Rates of processing determine the immunogenicity of immunoproteasomegenerated epitopes. J Immunol 178(12):7557-7562

48. Strehl B, Joeris T, Rieger M et al (2006) Immunoproteasomes are essential for clearance of Listeria monocytogenes in nonlymphoid tissues but not for induction of bacteria-specific CD8+ T cells. J Immunol 177(9):6238-6244

49. Kuckelkorn U, Ruppert T, Strehl B et al (2002) Link between organ-specific antigen processing by $20 \mathrm{~S}$ proteasomes and CD8(+) T cell-mediated autoimmunity. J Exp Med 195(8):983-990

50. Strehl B, Textoris-Taube K, Jakel S et al (2008) Antitopes define preferential proteasomal cleavage site usage. J Biol Chem 283(26):17891-17897
51. Cascio P, Hilton C, Kisselev AF et al (2001) 26S proteasomes and immunoproteasomes produce mainly $\mathrm{N}$-extended versions of an antigenic peptide. EMBO J 20(10):2357-2366

52. Beekman NJ, van Veelen PA, van Hall T et al (2000) Abrogation of CTL epitope processing by single amino acid substitution flanking the C-terminal proteasome cleavage site. J Immunol 164(4):1898-1905

53. Seifert U, Liermann H, Racanelli V et al (2004) Hepatitis C virus mutation affects proteasomal epitope processing. J Clin Invest 114(2):250-259

54. Ossendorp F, Eggers M, Neisig A et al (1996) A single residue exchange within a viral CTL epitope alters proteasome-mediated degradation resulting in lack of antigen presentation. Immunity 5(2):115-124

55. Tenzer S, Wee E, Burgevin A et al (2009) Antigen processing influences HIV-specific cytotoxic T lymphocyte immunodominance. Nat Immunol 10(6):636-646

56. Groettrup M, Soza A, Eggers M et al (1996) A role for the proteasome regulator PA28alpha in antigen presentation. Nature 381(6578):166-168

57. Dick TP, Ruppert T, Groettrup M et al (1996) Coordinated dual cleavages induced by the proteasome regulator PA28 lead to dominant MHC ligands. Cell 86(2):253-262

58. Sun Y, Sijts AJ, Song M et al (2002) Expression of the proteasome activator PA28 rescues the presentation of a cytotoxic T lymphocyte epitope on melanoma cells. Cancer Res 62(10): 2875-2882

59. Sijts A, Sun Y, Janek K et al (2002) The role of the proteasome activator PA28 in MHC class I antigen processing. Mol Immunol 39(3-4):165-169

60. Textoris-Taube K, Henklein P, Pollmann S et al (2007) The N-terminal flanking region of the TRP2360-368 melanoma antigen determines proteasome activator PA28 requirement for epitope liberation. J Biol Chem 282(17):12749-12754

61. van Hall T, Sijts A, Camps M et al (2000) Differential influence on cytotoxic $\mathrm{T}$ lymphocyte epitope presentation by controlled expression of either proteasome immunosubunits or PA28. J Exp Med 192(4):483-494

62. Schwarz K, Eggers M, Soza A et al (2000) The proteasome regulator PA28alpha/beta can enhance antigen presentation without affecting $20 \mathrm{~S}$ proteasome subunit composition. Eur J Immunol 30(12):3672-3679

63. Yamano T, Sugahara H, Mizukami S et al (2008) Allele-selective effect of PA28 in MHC class I antigen processing. J Immunol 181(3):1655-1664

64. Stohwasser R, Standera S, Peters I et al (1997) Molecular cloning of the mouse proteasome subunits MC14 and MECL-1: reciprocally regulated tissue expression of interferon-gammamodulated proteasome subunits. Eur J Immunol 27(5):11821187

65. Macagno A, Gilliet M, Sallusto F et al (1999) Dendritic cells upregulate immunoproteasomes and the proteasome regulator PA28 during maturation. Eur J Immunol 29(12):4037-4042

66. Li J, Schuler-Thurner B, Schuler G et al (2001) Bipartite regulation of different components of the MHC class I antigenprocessing machinery during dendritic cell maturation. Int Immunol 13(12):1515-1523

67. Macagno A, Kuehn L, de Giuli R, Groettrup M (2001) Pronounced up-regulation of the PA28alpha/beta proteasome regulator but little increase in the steady-state content of immunoproteasome during dendritic cell maturation. Eur J Immunol 31(11):3271-3280

68. Ossendorp F, Fu N, Camps M et al (2005) Differential expression regulation of the alpha and beta subunits of the PA28 proteasome activator in mature dendritic cells. J Immunol 174(12):7815-7822 
69. Basler M, Youhnovski N, Van Den Broek M et al (2004) Immunoproteasomes down-regulate presentation of a subdominant $\mathrm{T}$ cell epitope from lymphocytic choriomeningitis virus. J Immunol 173(6):3925-3934

70. Hensley SE, Zanker D, Dolan BP et al (2010) Unexpected role for the immunoproteasome subunit LMP2 in antiviral humoral and innate immune responses. J Immunol 184(8):4115-4122

71. Frisan T, Levitsky V, Polack A, Masucci MG (1998) Phenotypedependent differences in proteasome subunit composition and cleavage specificity in B cell lines. J Immunol 160(7):32813289

72. Frisan T, Levitsky V, Masucci MG (2000) Variations in proteasome subunit composition and enzymatic activity in B-lymphoma lines and normal B cells. Int J Cancer 88(6):881889

73. Murata S, Sasaki K, Kishimoto T et al (2007) Regulation of $\mathrm{CD} 8+\mathrm{T}$ cell development by thymus-specific proteasomes. Science 316(5829):1349-1353

74. Shin EC, Seifert U, Kato T et al (2006) Virus-induced type I IFN stimulates generation of immunoproteasomes at the site of infection. J Clin Invest 116(11):3006-3014

75. Barton LF, Cruz M, Rangwala R et al (2002) Regulation of immunoproteasome subunit expression in vivo following pathogenic fungal infection. J Immunol 169(6):3046-3052

76. Levitskaya J, Sharipo A, Leonchiks A et al (1997) Inhibition of ubiquitin/proteasome-dependent protein degradation by the GlyAla repeat domain of the Epstein-Barr virus nuclear antigen 1. Proc Natl Acad Sci USA 94(23):12616-12621

77. Wieland SF, Vega RG, Muller R et al (2003) Searching for interferon-induced genes that inhibit hepatitis $\mathrm{B}$ virus replication in transgenic mouse hepatocytes. J Virol 77(2):1227-1236

78. Khan S, van den Broek M, Schwarz K et al (2001) Immunoproteasomes largely replace constitutive proteasomes during an antiviral and antibacterial immune response in the liver. J Immunol 167(12):6859-6868

79. Chen W, Norbury CC, Cho Y et al (2001) Immunoproteasomes shape immunodominance hierarchies of antiviral CD8(+) T cells at the levels of $\mathrm{T}$ cell repertoire and presentation of viral antigens. J Exp Med 193(11):1319-1326

80. Pang KC, Sanders MT, Monaco JJ et al (2006) Immunoproteasome subunit deficiencies impact differentially on two immunodominant influenza virus-specific $\mathrm{CD} 8+\mathrm{T}$ cell responses. J Immunol 177(11):7680-7688

81. Robek MD, Garcia ML, Boyd BS, Chisari FV (2007) Role of immunoproteasome catalytic subunits in the immune response to hepatitis B virus. J Virol 81(2):483-491

82. Nussbaum AK, Rodriguez-Carreno MP, Benning N et al (2005) Immunoproteasome-deficient mice mount largely normal CD8+ $\mathrm{T}$ cell responses to lymphocytic choriomeningitis virus infection and DNA vaccination. J Immunol 175(2):1153-1160

83. Jakel S, Kuckelkorn U, Szalay G et al (2009) Differential interferon responses enhance viral epitope generation by myocardial immunoproteasomes in murine enterovirus myocarditis. Am J Pathol 175(2):510-518

84. Tu L, Moriya C, Imai T et al (2009) Critical role for the immunoproteasome subunit LMP7 in the resistance of mice to Toxoplasma gondii infection. Eur J Immunol 39(12):3385-3394

85. Schubert U, Anton LC, Gibbs J et al (2000) Rapid degradation of a large fraction of newly synthesized proteins by proteasomes. Nature 404(6779):770-774

86. Lelouard H, Ferrand V, Marguet D et al (2004) Dendritic cell aggresome-like induced structures are dedicated areas for ubiquitination and storage of newly synthesized defective proteins. J Cell Biol 164(5):667-675

87. Yewdell JW (2005) The seven dirty little secrets of major histocompatibility complex class I antigen processing. Immunol Rev 207(1):8-18

88. Vigneron N, Stroobant V, Chapiro J, Ooms A, Degiovanni G, Morel S, van der Bruggen P, Boon T, Van den Eynde BJ (2004) An antigenic peptide produced by peptide splicing in the proteasome. Science 304:587-590

89. Hanada K, Yewdell JW, Yang JC (2004) Immune recognition of a human renal cancer antigen through post-translational protein splicing. Nature 427(6971):252-256

90. Vigneron N, Stroobant V, Chapiro J et al (2004) An antigenic peptide produced by peptide splicing in the proteasome. Science 304(5670):587-590

91. Warren EH, Vigneron NJ, Gavin MA et al (2006) An antigen produced by splicing of noncontiguous peptides in the reverse order. Science 313(5792):1444-1447

92. Dalet A, Vigneron N, Stroobant V et al (2010) Splicing of distant peptide fragments occurs in the proteasome by transpeptidation and produces the spliced antigenic peptide derived from fibroblast growth factor-5. J Immunol 184(6):3016-3024

93. Basler M, Moebius J, Elenich L et al (2006) An altered T cell repertoire in MECL-1-deficient mice. J Immunol 176(11):66656672

94. Nitta T, Murata S, Sasaki K et al (2010) Thymoproteasome shapes immunocompetent repertoire of $\mathrm{CD} 8+\mathrm{T}$ cells. Immunity 32(1):29-40

95. Doherty PC, Topham DJ, Tripp RA et al (1997) Effector CD4+ and CD8+ T-cell mechanisms in the control of respiratory virus infections. Immunol Rev 159:105-117

96. Osterloh P, Linkemann K, Tenzer S et al (2006) Proteasomes shape the repertoire of $\mathrm{T}$ cells participating in antigen-specific immune responses. Proc Natl Acad Sci USA 103(13):50425047

97. Caudill CM, Jayarapu K, Elenich L et al (2006) T cells lacking immunoproteasome subunits MECL-1 and LMP7 hyperproliferate in response to polyclonal mitogens. J Immunol 176(7):4075-4082

98. Zaiss DM, de Graaf N, Sijts AJ (2008) The proteasome immunosubunit multicatalytic endopeptidase complex-like 1 is a T-cell-intrinsic factor influencing homeostatic expansion. Infect Immun 76(3):1207-1213

99. Muchamuel T, Basler M, Aujay MA et al (2009) A selective inhibitor of the immunoproteasome subunit LMP7 blocks cytokine production and attenuates progression of experimental arthritis. Nat Med 15(7):781-787

100. Dolan BP, Li L, Takeda K, Bennink JR, Yewdell JW (2010) Defective ribosomal products are the major source of antigenic peptides endogenously generated from influenza A virus neuraminidase. J Immunol 184:1419-1424

101. Vabulas RM, Hartl FU (2005) Protein synthesis upon acute nutrient restriction relies on proteasome function. Science 310(5756): 1960-1963

102. Yewdell JW, Nicchitta CV (2006) The DRiP hypothesis decennial: support, controversy, refinement and extension. Trends Immunol 27(8):368-373

103. Seifert U, Bialy LP, Ebstein F et al (2010) Immunoproteasomes preserve protein homeostasis upon interferon-induced oxidative stress. Cell 142(4):613-624 